\section{OPEN ACCESS}

Edited by:

Stephanie Ameis,

The Hospital for Sick Children and

University of Toronto, Canada

Reviewed by:

Rosana Lima Pagano,

Hospital Sírio-Libanês, Brazil

Mehereen Bhaijiwala,

The Hospital for Sick Children,

Canada

*Correspondence:

Shinichiro Nagamitsu,

Department of Pediatrics and Child Health, Kurume University School of Medicine, 67 Asahi-machi Kurume City, Fukuoka 830-0011, Japan kaoru@med.kurume-u.ac.jp

Specialty section:

This article was submitted to

Neuropsychiatric Imaging and Stimulation, a section of the journal Frontiers in Psychiatry

Received: 16 February 2015 Accepted: 18 May 2015 Published: 02 June 2015

Citation:

Nagamitsu S, Yamashita Y,

Tanigawa H, Chiba H, Kaida $\mathrm{H}$, Ishibashi M, Kakuma T, Croarkin PE and Matsuishi T (2015) Upregulated

GABA inhibitory function in $A D H D$

children with child behavior checklist-dysregulation profile: 1231-iomazenil SPECT study.

Front. Psychiatry 6:84. doi: 10.3389/fpsyt.2015.00084

\title{
Upregulated GABA inhibitory function in ADHD children with child behavior checklist-dysregulation profile: 123I-iomazenil SPECT study
}

\begin{abstract}
Shinichiro Nagamitsu ${ }^{1 *}$, Yushiro Yamashita ${ }^{1}$, Hitoshi Tanigawa ${ }^{2}$, Hiromi Chiba ${ }^{3}$, Hayato Kaida ${ }^{2}$, Masatoshi Ishibashi ${ }^{2}$, Tatsuyuki Kakuma ${ }^{4}$, Paul E. Croarkin ${ }^{5}$ and Toyojiro Matsuishi ${ }^{1}$
\end{abstract}

${ }^{1}$ Department of Pediatrics and Child Health, Kurume University School of Medicine, Fukuoka, Japan, ${ }^{2}$ Department of Radiology, Kurume University School of Medicine, Fukuoka, Japan, ${ }^{3}$ Department of Psychiatry, Kurume University School of Medicine, Fukuoka, Japan, ${ }^{4}$ Biostatistics Center, Kurume University School of Medicine, Fukuoka, Japan, ${ }^{5}$ Department of Psychiatry and Psychology, Mayo Clinic, Rochester, MN, USA

The child behavior checklist-dysregulation profile (CBCL-DP) refers to a pattern of elevated scores on the attention problems, aggression, and anxiety/depression subscales of the child behavior checklist. The aim of the present study was to investigate the potential role of GABA inhibitory neurons in children with attention deficit/hyperactivity disorder (ADHD) and dysregulation assessed with a dimensional measure. Brain single photon emission computed tomography (SPECT) was performed in 35 children with ADHD using 123I-iomazenil, which binds with high affinity to benzodiazepine receptors. lomazenil binding activities were assessed with respect to the presence or absence of a threshold CBCL-DP (a score $\geq 210$ for the sum of the three subscales: Attention Problems, Aggression, and Anxiety/Depression). We then attempted to identify which CBCL-DP subscale explained the most variance with respect to SPECT data, using "age," "sex," and "history of maltreatment" as covariates. Significantly higher iomazenil binding activity was seen in the posterior cingulate cortex (PCC) of ADHD children with a significant CBCL-DP. The Anxiety/Depression subscale on the CBCL had significant effects on higher iomazenil binding activity in the left superior frontal, middle frontal, and temporal regions, as well as in the PCC. The present brain SPECT findings suggest that GABAergic inhibitory neurons may play an important role in the neurobiology of the CBCL-DP, in children with ADHD.

Keywords: CBCL-dysregulation profile, iomazenil, GABA, ADHD

\section{Introduction}

Severe behavioral and affective dysregulation with symptoms, such as hyperactivity, aggression, irritability, mood instability, and anxiety, contribute to significant academic and psychosocial impairment in children. Some of these symptoms are consistent with attention deficit hyperactivity disorder (ADHD). ADHD is the most frequent neuropsychiatric disorder in children and often presents with co-occurring disruptive behavior disorders, anxiety disorders, and bipolar disorder. Hyperactivity, irritability, and impulsivity place children at risk of maltreatment as a result of 
strained parent-child interactions $(1,2)$. The insecure parent-child relationship further exacerbates the behavioral and affective dysregulation observed in children.

The child behavior checklist-dysregulation profile (CBCL-DP) refers to a pattern of elevated scores on the Attention Problems, Aggression, and Anxiety/Depression subscales of the child behavior checklist (CBCL) (3). The CBCL-DP was originally proposed as a means of identifying youth with bipolar disorder (4). However, recent studies suggest that the results of the CBCL-DP are not simply an early manifestation of a single disease process, but rather that the CBCL-DP can be used as a developmental risk marker for a persisting deficit in self-regulation of affect and behavior $(5,6)$. The CBCL-DP may be best interpreted as an indicator of symptom severity and functional impairment $(7,8)$. Children with ADHD who had a threshold level CBCL-DP score $(\geq 210)$ showed higher rates of comorbidity disorders, including oppositional defiant disorder (ODD), conduct disorder (CD), anxiety disorder, bipolar disorder, and depression (9). The CBCL-DP is also associated with mood, anxiety, disruptive behavior disorders, and substance use in adulthood (3).

The underlying neurobiological defects or aberrant neuronal activity leading to the dysregulation profile in children with ADHD are elusive. Reducing serotoninergic function in children with ADHD and a significant CBCL-DP resulted in slower cognitive performance compared to children with ADHD who did not have the CBCL-DP, indicating that serotoninergic function could play a decisive role in the etiology of the CBCL-DP (10). In addition, the CBCL subscale of "Aggression" was found to be the main discriminator of ADHD children with CBCL-DP versus those without CBCL-DP with respect to serotoninergic dysfunction. Conversely, prior translational work with magnetic resonance spectroscopy and transcanial magnetic stimulation paradigms suggest that GABAergic neurochemistry and neurotransmission are dysregulated in children with ADHD (11). Ongoing work also suggests that defects in the GABAergic system in adults increase an individual's vulnerability to severe psychiatric illnesses due to aberrant regulation of serotoninergic and/or dopaminergic neurons $(12,13)$. Previous biochemical and pharmacological studies indicate that deficits in GABA receptor function, induced by intravenous infusion of iomazenil followed by a serotoninergic agonist, predispose healthy volunteers to increased anxiety and dissociative disturbances, suggesting that deficits in the GABAergic system may contribute to the pathophysiology of serotonininduced psychosis (12).

123I-iomazenil is a radioactive ligand for central-type benzodiazepine receptors that forms a complex with GABA(A) receptors. Thus, 123I-iomazenil single photon emission computed tomography (SPECT) can indirectly index GABA receptor function. 123Iiomazenil is a frequently used radionuclide tracer for presurgical evaluation of patients with refractory partial epilepsy $(14,15)$. Moreover, recent neuroimaging studies have explored the role of GABAergic inhibitory function in psychiatric disorders such as schizophrenia, anxiety disorders, and developmental disorders (16-20). To our knowledge, there is no previous work which characterizes GABA receptor functioning with 123I-iomazenil SPECT among children with ADHD. The working hypothesis of the present study was that behavioral and affective symptoms in children with ADHD, reflected in CBCL-DP scores, would correlate with changes in cortical GABAergic neuronal activity. To confirm this hypothesis, brain SPECT was performed using 123Iiomazenil in ADHD children with or without CBCL-DP. Further, we tried to identify which of the three significant scales in the CBCL-DP explains the most variance with respect to SPECT data using "age," "sex," and "history of maltreatment" as covariates.

\section{Materials and Methods}

\section{Ethics Statement}

The design of the study and procedures for obtaining informed consent were approved by the Medical Ethics Committee of Kurume University School of Medicine (\#10081). Informed consent was obtained from each child and his/her parents prior to their participation in the study.

\section{Participants}

Thirty-five children with ADHD (23 boys, 12 girls) enrolled in the study. Participants were recruited after visits from the Department of Pediatrics, Kurume University, for the management of externalizing symptoms (e.g., difficulty maintaining attention, restlessness, hyperactivity, and aggressive behavior) or internalizing symptoms (e.g., anxiety, dissociation, and depressive symptoms). A diagnosis of ADHD was made using the Diagnostic and Statistical Manual of Mental Disorders, 4th Edition, Text Revision (Dsm-Iv-Tr) (21). Children who had anxious or depressive symptoms, but did not have ADHD symptoms were excluded in this study. Of the 35 child participants with ADHD, 15 had the combined type, 11 had hyperactive-impulsive type, and 9 had inattentive type. Seventeen children ( 7 male, 10 females) had experienced an obvious maltreatment, such as physical $(n=9)$, psychological $(n=6)$, or sexual abuse $(n=1)$, or sexual assault $(n=1)$ during preschool. In 11 of these instances, a child-welfare consultation center had previously supported the families in hopes of preventing maltreatment. Two children stayed in a child residential care institution. However, none of the participants met the diagnostic criteria for posttraumatic stress disorder (PTSD) on assessment. Seven of the 35 subjects (20\%) had comorbid disorders, such as $\operatorname{ODD}(n=2), \mathrm{CD}(n=1)$, anxiety disorder $(n=2)$, and depression $(n=2)$. The mean age of the children at the time of their hospital visit was 10.4 years. All participants were medication naïve prior to enrollment.

\section{Child Behavior Checklist}

Behavioral and psychiatric assessments of the children included the $\mathrm{CBCL}, \mathrm{ADHD}$ rating scale (hyperactivity/impulsive and inattention scores, as well as total score) $(22,23)$, the Child Depression Inventory (CDI), the Child Dissociative Checklist (CDC), and the Wechsler Intelligence Scale for Children (WISC-III). The CBCL was used to evaluate children's emotional and behavioral functioning, competencies, and social problems, with specific items evaluating internalizing and externalizing symptoms, as well as attention and thought problems. Items evaluating internalizing symptoms focus on withdrawal, somatic complaints, and anxiety/depression. Items evaluating externalizing symptoms focus on delinquent or aggressive behavior. The CBCL-DP refers to 
a pattern of elevated scores on the Attention Problems, Aggression, and Anxiety/Depression subscales of the CBCL. A threshold CBCL-DP was defined as a score $\geq 210$ for the sum of three subscales. (4) Physicians rated the participants using the ADHD rating scale, $\mathrm{CDI}$, and $\mathrm{CDC}$, and the parents rated their children using the CBCL.

\section{lomazenil Single Photon Emission Computed Tomography and Analysis of Regions of Interest}

All 35 children underwent iomazenil SPECT imaging of the brain. Briefly, children were injected intravenously with a bolus of 95-117 MBq 123I-iomazenil (Nihon Medi-Physics, Tokyo, Japan), which binds with high affinity to benzodiazepine receptors. The SPECT scan was performed $3 \mathrm{~h}$ after injection of the tracer, without any sedation, using a large field-of-view dual-detector camera and a computer system equipped with a low-energy, high-resolution, parallel-hole collimator. The dual detector camera rotated over $180^{\circ}$ in a circular orbit and in 32 steps of $40 \mathrm{~s}$ each to cover $360^{\circ}$ in about $22 \mathrm{~min}$. Brain magnetic resonance imaging (MRI) was performed using a superconducting magnet operating at $1.5 \mathrm{~T}$. For coregistered SPECT and MRI analysis, a method of image integration was applied using Fusion Viewer software (Nihon Medi-Physics) with a registration algorithm based on maximum mutual information (Figure 1). Subsequently, the cortical and subcortical regions of interest (ROIs) in the acquired SPECT data were defined. Using elliptical templates, the ROIs were placed over the following regions: the superior frontal, middle frontal, parietal, temporal, and occipital regions in each hemisphere; the midbrain; and the anterior and posterior cingulate cortex (ACC and PCC, respectively; Figure 1). Each relative iomazenil binding activity in ROIs was expressed as a ratio of that in the occipital cortex. As 123I-iomazenil affinity in the occipital region was maximum and stable in brain cortex, the occipital region was used as a reference (24).

\section{Data Analysis}

The differences of each CBCL subscale, ADHD-RS, CDI score, $\mathrm{CDC}$ score, and Intelligence scale between ADHD children with/without CBCL-DP were compared by student's $t$-test. We first analyzed correlations between the relative iomazenil binding activity expressed as a ratio in each ROI and psychometric profiles after controlling for the effects of age, sex, and history of maltreatment. Further, we compared iomazenil binding activity with respect to the presence or absence of a threshold CBCL-DP score in these children and tried to identify which of the three CBCL-DP subscales explained the most variance with respect to the SPECT data. Associations between each of the CBCL-DP subscales and iomazenil binding activity in each brain area were evaluated using liner regression models, with "age," "sex," and "history of maltreatment" as covariates.

\section{Results}

Behavioral and psychiatric assessments of the participants were shown in Table 1. Of the 35 participants, 15 had a threshold CBCL-DP score (i.e., a score $\geq 210$ ) and 20 had CBCL-DP scores $<210$. The group with threshold CBCL-DP scores had a lower

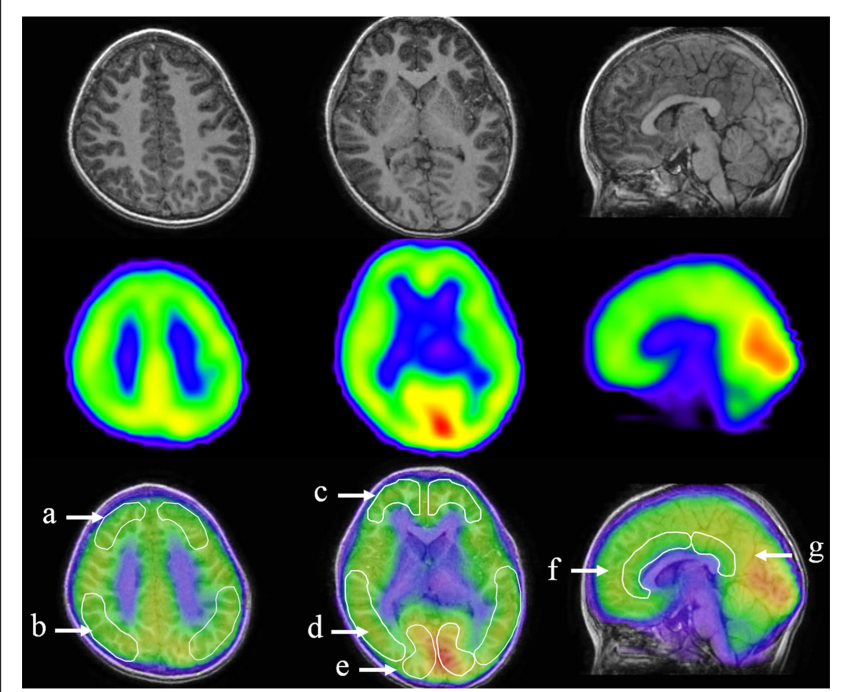

FIGURE 1 | Designated regions of interest (ROIs) in fusion images of 123I-iomazenil SPECT and MRI. The top panel shows brain MRI (transverse and sagittal $T_{1}$ sequences), the middle panel shows corresponding results of 123I-iomazenil SPECT, and the bottom panel shows fusion imaging. Outlined regions in the bottom panel indicate designated ROls, namely (a) the superior frontal, (b) parietal, (c) middle frontal,

(d) temporal, (e) occipital regions, (f) anterior, and (g) posterior cingulate gyrus.

ratio of male to female participants and more instances of maltreatment. Each ADHD rating scale, all subscales of CBCL with the exception of somatic problems, and CDC score were significantly higher in ADHD children with threshold CBCL-DP scores. Four participants with threshold level CBCL-DP scores had comorbidity disorders, including depression $(n=2)$, ODD $(n=1)$, and $\mathrm{CD}(n=1)$. Three participants without threshold CBCL-DP scores had comorbidity disorders, including anxiety disorder $(n=2)$ and ODD $(n=1)$. There was a difference in the CBCL-DP scores between participants with/without comorbidity disorders; however, this difference did not reach statistical significance $(n=28,199 \pm 20$, and $n=7,209 \pm 12$, respectively, $p=0.10)$.

Analyses of all participants $(n=35)$ revealed correlations between iomazenil binding activity in several brain regions and some part of the CBCL profile, after controlling for the effects of age, sex, and a history of maltreatment (Table 2). In both ACC and PCC, iomazenil binding activity had a statistically significant positive correlation with scores on the Anxiety/Depressed (Table 2; Figure 2), Internalizing, and Withdrawal Problems subscales of the CBCL (Table 2). In addition, significant positive correlations were noted for iomazenil binding activity in the ACC and Thought Problems on the CBCL, as well as for iomazenil binding activity in the PCC and Attention problems and Social Problems on the CBCL (Table 2). These significant correlations were not seen for other combinations in other brain regions, except for iomazenil binding activity in the midbrain and Thought Problems on the CBCL, and iomazenil binding activity in the right temporal region and Social Problems on the CBCL. There were no significant correlations between iomazenil binding activities in any brain region and any of the ADHD rating scales, CDI score, and CDC score 
TABLE 1 | Behavioral and psychiatric assessments of participants

\begin{tabular}{|c|c|c|}
\hline & $\begin{array}{c}\text { ADHD children } \\
\text { without significant } \\
\text { CBCL-DP }\end{array}$ & $\begin{array}{c}\text { ADHD children } \\
\text { with significant } \\
\text { CBCL-DP }\end{array}$ \\
\hline Number of participants & 20 & 15 \\
\hline Male:Female & $17 \pm 3$ & $6 \pm 9$ \\
\hline Mean age (years) & $10.7 \pm 1.9$ & $10.0 \pm 1.8$ \\
\hline Experience of maltreatment (\%) & 40 & 80 \\
\hline \multicolumn{3}{|l|}{ ADHD-RS } \\
\hline Total score & $23.5 \pm 9.2$ & $30.6 \pm 9.0^{*}$ \\
\hline Inattention score & $14.8 \pm 5.7$ & $17.9 \pm 4.9^{*}$ \\
\hline Impulsivity/hyperactivity score & $9.2 \pm 5.6$ & $12.6 \pm 6.0^{*}$ \\
\hline \multicolumn{3}{|l|}{ CBCL score } \\
\hline Internalizing score & $64.4 \pm 9.7$ & $80.8 \pm 8.2^{\star \star}$ \\
\hline Externalizing score & $62.7 \pm 8.5$ & $71.1 \pm 4.8^{\star \star}$ \\
\hline Aggressive behaviors & $63.7 \pm 9.0$ & $79.2 \pm 7.2^{\star \star}$ \\
\hline Anxious/depressed & $58.1 \pm 4.9$ & $66.4 \pm 4.5^{\star \star}$ \\
\hline Attention problem & $66.2 \pm 5.4$ & $73.7 \pm 5.5^{\star \star}$ \\
\hline Delinquent behavior & $62.7 \pm 8.5$ & $71.1 \pm 4.8^{\star \star}$ \\
\hline Withdrawn & $61.5 \pm 5.6$ & $66.3 \pm 5.6^{\star \star}$ \\
\hline Somatic problems & $55.5 \pm 15.2$ & $58.4 \pm 9.5$ \\
\hline Social problems & $62.0 \pm 8.8$ & $66.9 \pm 8.1^{*}$ \\
\hline Thought problems & $59.9 \pm 9.7$ & $67.8 \pm 9.9^{\star}$ \\
\hline CDI score & $14.3 \pm 9.6$ & $15.6 \pm 6.3$ \\
\hline CDC score & $6.7 \pm 3.8$ & $13.3 \pm 4.7^{\star \star}$ \\
\hline WISC-III & $90.0 \pm 15.6$ & $87.3 \pm 13.7$ \\
\hline
\end{tabular}

$C B C L-D P$, child behavior checklist-dysregulation profile; $A D H D-R S$, attention deficit hyperactivity disorder rating scale; CDI, child depression inventor; $C D C$, children dissociative checklist; WISC, Wechsler intelligence scale for children.

Significant difference from children without significant CBCL-DP (*indicates $p<0.05$,

**indicates $p<0.001$ ).

(data not shown). Iomazenil binding activity in the PCC was significantly higher in ADHD children with a threshold CBCL-DP score than in ADHD children with scores $<210$ after controlling for the effects of age, sex, and a history of maltreatment (Table 3, $F$-value $=4.36, p<0.05)$. Of the three CBCL-DP subscales, the Anxiety/Depression subscale had significant effects on higher iomazenil binding activity in the left superior frontal, middle frontal, and temporal regions, as well as in the PCC (Table 4).

\section{Discussion}

This is the first neuroimaging study showing that behavioral and affective symptoms in children with ADHD, reflected in CBCL-DP scores, are correlated with changes in cortical GABAergic neuronal activity. Overall, increased iomazenil activity in the ACC and PCC was associated with higher scores on many of the CBCL subscales. In ADHD children with a significant $\mathrm{CBCL}-\mathrm{DP}$, iomazenil activity was upregulated in the PCC. Of the three CBCL-DP subscales, the Anxiety/Depression subscale had a significant effect on iomazenil binding activity in many brain regions. These results suggest that behavioral and affective dysregulation in ADHD children may be characterized by changes of GABAergic neural activity. In this section, we discuss the role of the cingulate cortex in GABA function, the association between CBCL-DP scores and GABA function, and age-dependent differences in GABA function.

The cingulate cortex is one of the largest parts of the limbic lobe and the prefronto-limbic circuitry. The ACC plays key roles in emotion, motivation, and motor functions, whereas the PCC is involved in emotion, facial recognition, and memory functions (25-27). In the present study, we found higher iomazenil binding activity in ACC and PCC that was associated with higher scores on many of the CBCL subscales in ADHD children with and without CBCL-DP. Similar findings have been reported in healthy adults. For example, Kim et al. (28) found a positive correlation in healthy subjects between high GABA concentrations in the ACC and a high harm avoidance temperament, characterized by worrying about potential problems, fearful of uncertainties, and being shy in unfamiliar environments. Moreover, increased activity in the PCC has been observed in emotional disorders, including obsessive-compulsive disorder, major depression, and social phobia $(29,30)$. Because the cingulate cortex has been suggested to have an important role in modulating human fear and anxiety by modulating the activity of other limbic structures, including the amygdala (31), the increased GABAergic function in the cingulate cortex of ADHD children in the present study may have inhibited excessive excitation of the limbic system, which contributes to the development of behavioral and affective dysregulation.

We found that the Anxiety/Depression subscale of the CBCL-DP explains the most variance with respect to SPECT data in various brain regions using "age," "sex," and "history of maltreatment" as covariates. The Aggression and Attention Problem subscales of the CBCL-DP had no significant effects on SPECT data in various brain regions. These findings strongly support previous converging lines of evidence regarding the association between GABAergic activation and increased anxiety (32). Conversely, several biochemical and genetic studies have provided evidence of a significant role of serotoninergic function in aggressive behavior. For example, an inverse correlation has been reported between downregulated platelet or CSF 5-hydroxyindolecetic acid (5-HIAA), a major metabolite of serotonin, and levels of aggression and impulsivity $(33,34)$. Furthermore, Haberstick et al. (35) reported an association between certain promoter polymorphisms in the serotonin transporter (5HTTLPR) and greater aggressive behavior in middle childhood, suggesting that differences in serotonergic functioning may be a contributing factor to different levels of aggressive behavior. In terms of the biological mechanism underlying attention function, an important role for dopaminergic neurons has been proposed. Several neuroimaging studies have shown aberrant dopamine transporter (DAT) levels in the nucleus accumbens, caudate, and midbrain, as well as a positive relationship between DAT levels in the putamen and inattention scores in ADHD patients (36-38). Together, these findings suggest that changes in several neurotransmitter systems, including serotoninergic, dopaminergic, and GABAergic neurons, are likely to be involved in constructing the clinical manifestations of the CBCL-DP.

Significant positive correlations between GABAergic inhibitory function and the Anxiety/Depression subscale were also seen in our study. Although previous neuroimaging studies have reported those correlations in adulthood with psychiatric disorders $(17,28)$, the present study is the first report of the correlation in childhood with psychiatric disorders. Despite the positive correlation in childhood, previous neuroimaging studies using iomazenil SPECT have revealed negative correlations between 
TABLE 2 | Partial correlation coefficients between CBCL profiles and the iomazenil binding activites in each brain region

\begin{tabular}{|c|c|c|c|c|c|c|c|c|c|c|c|}
\hline & \multicolumn{2}{|c|}{ Superior frontal } & \multicolumn{2}{|c|}{ Parietal } & \multicolumn{2}{|c|}{ Middle frontal } & \multicolumn{2}{|c|}{ Temporal } & \multirow[t]{2}{*}{ Mid brain } & \multirow[t]{2}{*}{ ACC } & \multirow[t]{2}{*}{ PCC } \\
\hline & $\mathbf{R}$ & $\mathbf{L}$ & $\mathbf{R}$ & $\mathbf{L}$ & $\mathbf{R}$ & $\mathbf{L}$ & $\mathbf{R}$ & $\mathbf{L}$ & & & \\
\hline \multicolumn{12}{|l|}{ CBCL profiles } \\
\hline Total problems & 0.341 & 0.237 & 0.192 & 0.267 & 0.301 & 0.229 & 0.284 & 0.191 & 0.178 & 0.385 & 0.245 \\
\hline Internalizing problems & 0.199 & 0.160 & 0.026 & 0.068 & 0.110 & 0.188 & 0.136 & 0.332 & 0.274 & $0.477^{\star}$ & $0.536^{\star \star}$ \\
\hline Externalizing problems & 0.158 & 0.086 & 0.057 & 0.090 & 0.148 & 0.027 & 0.127 & -0.072 & 0.032 & 0.078 & -0.041 \\
\hline Aggressive behaviors $^{a}$ & 0.119 & 0.052 & 0.036 & 0.077 & 0.113 & -0.042 & 0.088 & -0.111 & -0.040 & 0.017 & -0.026 \\
\hline Anxious/depressed ${ }^{\mathrm{a}}$ & 0.309 & 0.284 & 0.120 & 0.129 & 0.264 & 0.335 & 0.246 & 0.369 & 0.355 & $0.536^{\star \star}$ & $0.524^{\star \star}$ \\
\hline Attention problem ${ }^{\mathrm{a}}$ & 0.265 & 0.120 & 0.234 & 0.157 & 0.228 & 0.166 & 0.248 & 0.160 & 0.212 & 0.378 & $0.483^{\star \star}$ \\
\hline Delinquent behavior & 0.122 & 0.091 & 0.068 & 0.102 & 0.133 & 0.087 & 0.175 & 0.108 & 0.159 & 0.077 & 0.008 \\
\hline Withdrawn & 0.278 & 0.105 & 0.163 & 0.006 & 0.192 & 0.057 & 0.338 & 0.214 & -0.012 & $0.425^{\star}$ & $0.509^{\star *}$ \\
\hline Somatic problems & -0.131 & 0.045 & -0.162 & 0.035 & -0.133 & 0.074 & -0.246 & 0.080 & 0.216 & -0.050 & 0.205 \\
\hline Social problems & 0.304 & 0.112 & 0.325 & 0.217 & 0.264 & 0.108 & $0.406^{\star}$ & 0.204 & 0.129 & 0.383 & $0.440^{\star}$ \\
\hline Thought problems & 0.322 & 0.221 & 0.269 & 0.207 & 0.307 & 0.320 & 0.310 & 0.239 & $0.546^{\star \star}$ & $0.432^{\star}$ & 0.360 \\
\hline
\end{tabular}

$C B C L$, child behavior checklist; ACC, anterior cingulate cortex; PCC, posterior cingulate cortex; $R$, right; $L$, left.

*Indicates $p<0.05$; **indicates $p<0.01$.

alndicates subscale which comprises CBCL-DP (dysregulation profile).

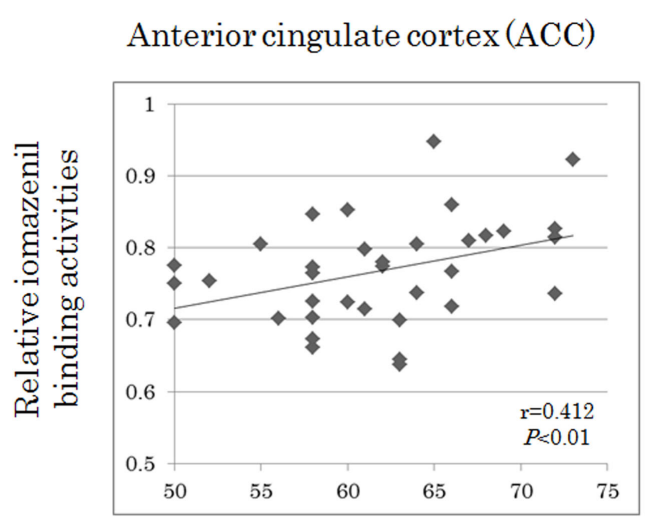

\section{Posterior cingulate cortex (PCC)}

Score of subscale CBCL Anxiety/Depressed

FIGURE 2 | Correlations between iomazenil binding activity in the anterior and posterior cingulate cortices and score of subscale CBCL anxiety/depressed in all subjects. The higher relative iomazenil

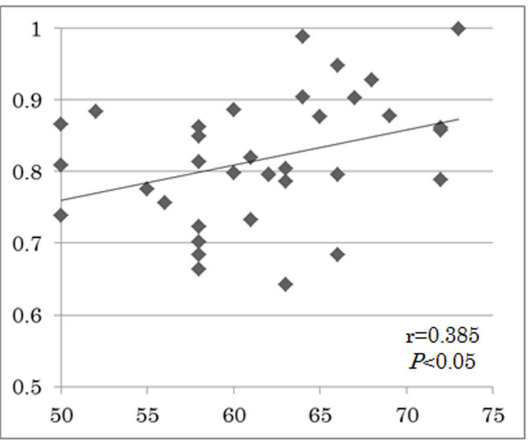

binding activities in ACC and PCC are significantly associated with the higher scores of "Anxiety/Depressed" subscale in the CBCL. CBCL, child behavior checklist.
TABLE 3 | Effect of the significant CBCL-DP (score $\geq 210$ ) on iomazenil binding activities in each brain region.

\begin{tabular}{lllr}
\hline Brain area & \multicolumn{1}{c}{ Effect } & $\boldsymbol{F}$-Test & p-Value \\
\hline Right superior frontal & Significant CBCL-DP & 0.88 & 0.35 \\
Left superior frontal & Significant CBCL-DP & 0.18 & 0.67 \\
Right parietal & Significant CBCL-DP & 2.07 & 0.16 \\
Left parietal & Significant CBCL-DP & 0.37 & 0.55 \\
Right middle frontal & Significant CBCL-DP & 1.57 & 0.22 \\
Left middle frontal & Significant CBCL-DP & 0.44 & 0.51 \\
Right temporal & Significant CBCL-DP & 1.26 & 0.27 \\
Left temporal & Significant CBCL-DP & 0.05 & 0.82 \\
Midbrain & Significant CBCL-DP & 0.2 & 0.66 \\
Anterior cingulate cortex & Significant CBCL-DP & 0.99 & 0.33 \\
Posterior cingulate cortex & Significant CBCL-DP & 4.36 & $<0.05$ \\
\hline
\end{tabular}

$C B C L-D P$, child behavior checklist-dysregulation profile.

GABA-benzodiazepine receptor binding activity and the severity of anxiety symptoms in adults with panic or traumatic disorders (17-19). It is well known that there are considerable changes in the number of GABA receptors and in subunit expression during brain development (39). Specifically, the greatest number of GABA receptors is found in the youngest children, with numbers decreasing exponentially with age, and there are age-related increases in $\alpha 1$-subunit-containing GABA receptors (40). These age-related changes in GABA receptors may affect outcomes when assessing increases and/or decreases in overall iomazenil binding activity in children.

The present study has several limitations that require consideration in future studies. For example, in the present study, SPECT exhibited poor resolution around some limbic regions, such as the amygdala and hippocampus, which are important for emotion processing. In these small regions, the obtained radioactivity might differ from the true activity because of partial volume effect (PVE). The PVE can be defined as the underestimation of binding per unit brain volume in small objects or regions because of the blurring of the radioactivity (spillout and spill-in) between regions. These regions need to be resolved using MR imaging-based correction for PVE (41, 42). Brain imaging data from normal healthy children are not available 
TABLE 4 | Effect of the CBCL-DP subscale on iomazenil binding activities in each brain region.

\begin{tabular}{|c|c|c|c|}
\hline Brain area & Effect & $F$-Test & $p$-Value \\
\hline \multirow[t]{3}{*}{ Right superior frontal } & Aggressive behavior & 0.03 & 0.8708 \\
\hline & Anxious/depressed & 2.79 & 0.1058 \\
\hline & Attention problem & 0.12 & 0.7268 \\
\hline \multirow[t]{3}{*}{ Left superior frontal } & Aggressive behavior & 0.01 & 0.9374 \\
\hline & Anxious/depressed & 4.28 & $0.0479^{*}$ \\
\hline & Attention problem & 1.6 & 0.2157 \\
\hline \multirow[t]{3}{*}{ Right parietal } & Aggressive behavior & 0 & 0.9675 \\
\hline & Anxious/depressed & 0.13 & 0.7249 \\
\hline & Attention problem & 0.39 & 0.5367 \\
\hline \multirow[t]{3}{*}{ Left parietal } & Aggressive behavior & 0.33 & 0.5703 \\
\hline & Anxious/depressed & 0.67 & 0.4214 \\
\hline & Attention problem & 0.02 & 0.8843 \\
\hline \multirow[t]{3}{*}{ Right middle frontal } & Aggressive behavior & 0 & 0.9771 \\
\hline & Anxious/depressed & 1.83 & 0.1871 \\
\hline & Attention problem & 0.06 & 0.8060 \\
\hline \multirow[t]{3}{*}{ Left middle frontal } & Aggressive behavior & 0.5 & 0.4873 \\
\hline & Anxious/depressed & 5.1 & $0.0319^{*}$ \\
\hline & Attention problem & 0.92 & 0.3457 \\
\hline \multirow[t]{3}{*}{ Right temporal } & Aggressive behavior & 0.03 & 0.8709 \\
\hline & Anxious/depressed & 1.46 & 0.2363 \\
\hline & Attention problem & 0.02 & 0.8888 \\
\hline \multirow[t]{3}{*}{ Left temporal } & Aggressive behavior & 0.12 & 0.7354 \\
\hline & Anxious/depressed & 7.55 & $0.0104^{\star}$ \\
\hline & Attention problem & 1.91 & 0.1777 \\
\hline \multirow[t]{3}{*}{ Mid brain } & Aggressive behavior & 0.66 & 0.4231 \\
\hline & Anxious/depressed & 1.98 & 0.1699 \\
\hline & Attention problem & 0.21 & 0.6482 \\
\hline \multirow[t]{3}{*}{ Anterior cingulate cortex } & Aggressive behavior & 0.97 & 0.3331 \\
\hline & Anxious/depressed & 3.94 & 0.0570 \\
\hline & Attention problem & 1.06 & 0.3118 \\
\hline \multirow[t]{3}{*}{ Posterior cingulate cortex } & Aggressive behavior & 0.99 & 0.3277 \\
\hline & Anxious/depressed & 5.62 & $0.0248^{\star}$ \\
\hline & Attention problem & 1.26 & 0.2714 \\
\hline
\end{tabular}

$C B C L-D P$, child behavior checklist-dysregulation profile.

*Indicates significant effects on higher iomazenil binding activity.

because of ethical concerns with SPECT studies of this population. Therefore, we focused our research questions on the correlation between GABAergic inhibitory function in specific brain regions and psychometric profiles. It is possible that, in addition to the

\section{References}

1. Weinstein D, Staffelbach D, Biaggio M. Attention-deficit hyperactivity disorder and posttraumatic stress disorder: differential diagnosis in childhood sexual abuse. Clin Psychol Rev (2000) 20:359-78. doi:10.1016/S0272-7358(98) 00107-X

2. Ouyang L, Fang X, Mercy J, Perou R, Grosse SD. Attention-deficit/hyperactivity disorder symptoms and child maltreatment: a population-based study. J Pediatr (2008) 153:851-6. doi:10.1016/j.jpeds.2008.06.002

3. Althoff RR, Verhulst FC, Rettew DC, Hudziak JJ, van der Ende J. Adult outcomes of childhood dysregulation: a 14-year follow-up study. J Am Acad Child Adolesc Psychiatry (2010) 49:1105-16. doi:10.1016/j.jaac.2010.08.006

4. Biederman J, Petty CR, Monuteaux MC, Evans M, Parcell T, Faraone SV, et al. The child behavior checklist-pediatric bipolar disorder profile predicts a subsequent diagnosis of bipolar disorder and associated impairments in ADHD youth growing up: a longitudinal analysis. J Clin Psychiatry (2009) 70:732-40. doi:10.4088/JCP.08m04821

5. Holtmann M, Buchmann AF, Esser G, Schmidt MH, Banaschewski T, Laucht M. The child behavior checklist-dysregulation profile predicts substance use, population of people with $\mathrm{ADHD}$, our result is generalizable to the normal population. Furthermore, we selected iomazenil activity in the occipital regions as a reference, meaning that we could not evaluate inhibitory function in occipital regions. We did not clarify how putative dopaminergic or serotoninergic changes are involved in other subscales, such as the Aggression and Attention Problem subscales, of the CBCL-DP. It is possible that investigations incorporating the simultaneous assessment of benzodiazepine receptor binding activity and homovanillic acid (HVA) and 5HIAA in the urine (principal metabolites of dopamine and serotonin, respectively) could provide new insights into the underlying neurobiological defects or aberrant neuronal activity leading to the dysregulation profile in children.

In conclusion, the present 123I-iomazenil brain SPECT study provides evidence that changes in GABAergic inhibitory neuronal activity correlate with some elements of function measured by the CBCL-DP. Brain SPECT may be useful for the evaluation of the possible pathogenesis of neuropsychiatric symptoms observed in children.

\section{Author Contributions}

SN participated in the design of this study and compiled the manuscript. SN, YY, and HC saw the patients and obtained informed consent and their agreement to participate in the study. Diagnosis of comorbidity disorders was made by HC. SN and HC summarized participant behavioral and psychiatric assessments, including CBCL-DP data. Three radiologists (HT, HK, and MI) were in charge of radioactive measurements and calculations of iomazenil activity using ROIs. TK, a statistician, conducted the statistical analyses. PC and TM supervised the preparation of the manuscript.

\section{Acknowledgments}

This work was supported by grants from the Ministry of Education, Culture, Sports, Science, and Technology (\#22591143, \#25460643).

suicidality, and functional impairment: a longitudinal analysis. J Child Psychol Psychiatry (2011) 52:139-47. doi:10.1111/j.1469-7610.2010.02309.x

6. Meyer SE, Carlson GA, Youngstrom E, Ronsaville DS, Martinez PE, Gold PW, et al. Long-term outcomes of youth who manifested the CBCL-pediatric bipolar disorder phenotype during childhood and/or adolescence. J Affect Disord (2009) 113:227-35. doi:10.1016/j.jad.2008.05.024

7. Peyre H, Speranza M, Cortese S, Wohl M, Purper-Ouakil D. Do ADHD children with and without child behavior checklist-dysregulation profile have different clinical characteristics, cognitive features, and treatment outcomes? J Atten Disord (2015) 19:63-71. doi:10.1177/1087054712452135

8. McGough JJ, McCracken JT, Cho AL, Castelo E, Sturm A, Cowen J, et al A potential electroencephalography and cognitive biosignature for the child behavior checklist-dysregulation profile. J Am Acad Child Adolesc Psychiatry (2013) 52:1173-82. doi:10.1016/j.jaac.2013.08.002

9. Biederman J, Petty CR, Day H, Goldin RL, Spencer T, Faraone SV, et al. Severity of the aggression/anxiety-depression/attention child behavior checklist profile discriminates between different levels of deficits in emotional regulation in youth with attention-deficit hyperactivity disorder. J Dev Behav Pediatr (2012) 33:236-43. doi:10.1097/DBP.0b013e3182475267 
10. Zepf FD, Wöckel L, Poustka F, Holtmann M. Diminished 5-HT functioning in CBCL pediatric bipolar disorder-profiled ADHD patients versus normal ADHD: susceptibility to rapid tryptophan depletion influences reaction time performance. Hum Psychopharmacol (2008) 23:291-9. doi:10.1002/hup.934

11. Edden RA, Crocetti D, Zhu H, Gilbert DL, Mostofsky SH. Reduced GABA concentration in attention-deficit/hyperactivity disorder. Arch Gen Psychiatry (2012) 69:750-3. doi:10.1001/archgenpsychiatry.2011.2280

12. D’Souza DC, Gil RB, Zuzarte E, MacDougall LM, Donahue L, Ebersole JS, et al. Gamma-aminobutyric acid-serotonin interactions in healthy men: implications for network models of psychosis and dissociation. Biol Psychiatry (2006) 59:128-37. doi:10.1016/j.biopsych.2005.06.020

13. Scheel-Krüger J. Dopamine-GABA interactions: evidence that GABA transmits, modulates and mediates dopaminergic functions in the basal ganglia and the limbic system. Acta Neurol Scand Suppl (1986) 107:1-54.

14. Higurashi N, Hamano S, Oritsu T, Minamitani M, Sasaki M, Ida H. Iomazenil hyperfixation in single photon emission computed tomography study of malformations of cortical development during infancy. Eur J Paediatr Neurol (2011) 15:372-5. doi:10.1016/j.ejpn.2011.03.007

15. Kuroda H, Ogasawara K, Aso K, Beppu T, Kobayashi M, Chida K, et al. Spontaneous recovery of reduced cortical central benzodiazepine receptor binding potential on I-123 Iomazenil SPECT in a patient with status epilepticus. Clin Nucl Med (2010) 35:126-7. doi:10.1097/RLU.0b013e3181c7c168

16. Verhoeff NP, Soares JC, D'Souza CD, Gil R, Degen K, Abi-Dargham A, et al. $\left[{ }^{123} \mathrm{I}\right]$ Iomazenil SPECT benzodiazepine receptor imaging in schizophrenia. Psychiatry Res (1999) 91:163-73. doi:10.1016/S0925-4927(99)00027-X

17. Geuze E, van Berckel BN, Lammertsma AA, Boellaard R, de Kloet CS, Vermetten E, et al. Reduced GABAA benzodiazepine receptor binding in veterans with post-traumatic stress disorder. Mol Psychiatry (2008) 13:74-8. doi:10.1038/sj. mp.4002054

18. Hasler G, Nugent AC, Carlson PJ, Carson RE, Geraci M, Drevets WC. Altered cerebral gamma-aminobutyric acid type A-benzodiazepine receptor binding in panic disorder determined by $\left[{ }^{11} \mathrm{C}\right]$ flumazenil positron emission tomography. Arch Gen Psychiatry (2008) 65:1166-75. doi:10.1001/archpsyc.65.10.1166

19. Bremner JD, Innis RB, Southwick SM, Staib L, Zoghbi S, Charney DS. Decreased benzodiazepine receptor binding in prefrontal cortex in combat-related posttraumatic stress disorder. Am J Psychiatry (2000) 157:1120-6. doi:10.1176/appi. ajp.157.7.1120

20. Mori T, Mori K, Fujii E, Toda Y, Miyazaki M, Harada M, et al. Evaluation of the GABAergic nervous system in autistic brain: (123)I-iomazenil SPECT study. Brain Dev (2012) 34:648-54. doi:10.1016/j.braindev.2011.10.007

21. American Psychiatric Association. Diagnostic and Statistical Manual of Mental Disorders. 4th ed. Text Revision (DSM-IV-TR). Washington: American Psychiatric Association (2000).

22. Achenbach TM. Manual for the Child Behavior Checklist/4-18 and 1991 Child Profile. Burlington: University of Vermont Department of Psychiatry (1991).

23. DuPaul GJ, Anastopoulos AD, Power TJ, Reid R, Ikeda MJ, McGoey K. Parent ratings of attention-deficit/hyperactivity disorder symptoms: factor structure and normative data. J Psychopathol Behav Assess (1998) 20:83-102. doi:10.1023/ A:1023087410712

24. Laruelle M, Abi-Dargham A, Rattner Z, Al-Tikriti MS, Zea-Ponce Y, Zoghbi SS, et al. Single photon emission tomography measurement of benzodiazepine receptor number and affinity in primate brain: a constant infusion paradigm with [123I]iomazenil. Eur J Pharmacol (1993) 230:119-23. doi:10. 1016/0014-2999(93)90421-D

25. Maddock RJ, Garrett AS, Buonocore MH. Posterior cingulate cortex activation by emotional words: fMRI evidence from a valence decision task. Hum Brain Mapp (2003) 18:30-41. doi:10.1002/hbm.10075

26. Mega MS, Cummings JL. Frontal subcortical circuits. In: Salloway SP, Malloy PF, Duffy JD, editors. The Frontal Lobes and Neuropsychiatrie Illness. Washington: American Psychiatric Publishing (2001). p. 15-32.

27. Devinsky O, Morrell MJ, Vogt BA. Contributions of anterior cingulate cortex to behaviour. Brain (1995) 118:279-306. doi:10.1093/brain/118.1.279

28. Kim HJ, Kim JE, Cho G, Song IC, Bae S, Hong SJ, et al. Associations between anterior cingulate cortex glutamate and gamma-aminobutyric acid concentrations and the harm avoidance temperament. Neurosci Lett (2009) 464:103-7. doi:10.1016/j.neulet.2009.07.087
29. Bench CJ, Friston KJ, Brown RG, Scott LC, Frackowiak RS, Dolan RJ. The anatomy of melancholia - focal abnormalities of cerebral blood flow in major depression. Psychol Med (1992) 22:607-15. doi:10.1017/S003329170003806X

30. McGuire PK, Bench CJ, Frith CD, Marks IM, Frackowiak RS, Dolan RJ. Functional anatomy of obsessive-compulsive phenomena. Br J Psychiatry (1994) 164:459-68. doi:10.1192/bjp.164.4.459

31. Hahn A, Stein P, Windischberger C, Weissenbacher A, Spindelegger C, Moser $\mathrm{E}$, et al. Reduced resting-state functional connectivity between amygdala and orbitofrontal cortex in social anxiety disorder. Neuroimage (2011) 56:881-9. doi:10.1016/j.neuroimage.2011.02.064

32. Shen Q, Fuchs T, Sahir N, Luscher B. GABAergic control of critical developmental periods for anxiety- and depression-related behavior in mice. PLoS One (2012) 7:e47441. doi:10.1371/journal.pone.0047441

33. Kruesi MJ, Hibbs ED, Zahn TP, Keysor CS, Hamburger SD, Bartko JJ, et al. A 2-year prospective follow-up study of children and adolescents with disruptive behavior disorders. Prediction by cerebrospinal fluid 5-hydroxyindoleacetic acid, homovanillic acid, and autonomic measures? Arch Gen Psychiatry (1992) 49:429-35. doi:10.1001/archpsyc.1992.01820060009001

34. Golubchik P, Mozes T, Vered Y, Weizman A. Platelet poor plasma serotonin level in delinquent adolescents diagnosed with conduct disorder. Prog Neuropsychopharmacol Biol Psychiatry (2009) 33:1223-5. doi:10.1016/j.pnpbp. 2009.07.003

35. Haberstick BC, Smolen A, Hewitt JK. Family-based association test of the 5HTTLPR and aggressive behavior in a general population sample of children. Biol Psychiatry (2006) 59:836-43. doi:10.1016/j.biopsych.2005.10.008

36. Jucaite A, Fernell E, Halldin C, Forssberg H, Farde L. Reduced midbrain dopamine transporter binding in male adolescents with attentiondeficit/hyperactivity disorder: association between striatal dopamine markers and motor hyperactivity. Biol Psychiatry (2005) 57:229-38. doi:10.1016/j. biopsych.2004.11.009

37. Spencer TJ, Biederman J, Madras BK, Dougherty DD, Bonab AA, Livni E, et al. Further evidence of dopamine transporter dysregulation in ADHD: a controlled PET imaging study using altropane. Biol Psychiatry (2007) 62:1059-61. doi:10. 1016/j.biopsych.2006.12.008

38. da Silva N Jr, Szobot CM, Anselmi CE, Jackowski AP, Chi SM, Hoexter MQ, et al. Attention deficit/hyperactivity disorder: is there a correlation between dopamine transporter density and cerebral blood flow? Clin Nucl Med (2011) 36:656-60. doi:10.1097/RLU.0b013e318219b49d

39. Rissman RA, De Blas AL, Armstrong DM. GABA(A) receptors in aging and Alzheimer's disease. J Neurochem (2007) 103:1285-92. doi:10.1111/j. 1471-4159.2007.04832.x

40. Chugani DC, Muzik O, Juhász C, Janisse JJ, Ager J, Chugani HT. Postnatal maturation of human GABAA receptors measured with positron emission tomography. Ann Neurol (2001) 49:618-26. doi:10.1002/ana.1003

41. Kato H, Matsuda K, Baba K, Shimosegawa E, Isohashi K, Imaizumi M, et al. MR imaging-based correction for partial volume effect improves detectability of intractable epileptogenic foci on iodine 123 iomazenil brain SPECT images: an extended study with a larger sample size. AJNR Am J Neuroradiol (2012) 33:2088-94. doi:10.3174/ajnr.A3121

42. Kato H, Shimosegawa E, Oku N, Kitagawa K, Kishima H, Saitoh Y, et al. MRIbased correction for partial-volume effect improves detectability of intractable epileptogenic foci on 123I-iomazenil brain SPECT images. J Nucl Med (2008) 49:383-9. doi:10.2967/jnumed.107.046136

Conflict of Interest Statement: The authors declare that the research was conducted in the absence of any commercial or financial relationships that could be construed as a potential conflict of interest.

Copyright (C) 2015 Nagamitsu, Yamashita, Tanigawa, Chiba, Kaida, Ishibashi, Kakuma, Croarkin and Matsuishi. This is an open-access article distributed under the terms of the Creative Commons Attribution License (CC BY). The use, distribution or reproduction in other forums is permitted, provided the original author(s) or licensor are credited and that the original publication in this journal is cited, in accordance with accepted academic practice. No use, distribution or reproduction is permitted which does not comply with these terms. 